\title{
उмR
}

\section{HLA-A gene polymorphisms contribute to osteoporosis susceptibility in postmenopausal Han Chinese women}

\author{
S.M. Li ${ }^{1}$, H. Guo ${ }^{2}$, H.J. Yang ${ }^{1}$, M.Q. Lv ${ }^{3}$ and D.X. Zhou ${ }^{3,4}$ \\ ${ }^{1}$ Department of Orthopedics, \\ The First Affiliated Hospital of Xi'an Jiaotong University, Xi'an, China \\ ${ }^{2}$ Department of Emergency, \\ Xi'an Jiaotong University Medical College Affiliated Red Cross Hospital, \\ Xi'an, China \\ ${ }^{3}$ Department of Pathology, Medical School, Xi'an Jiaotong University, Xi'an, \\ China \\ ${ }^{4}$ Key Laboratory of Environment and Genes Related to Diseases, \\ Ministry of Education, Xi'an, China \\ Corresponding author: D.X. Zhou \\ E-mail: zhoudx2010@163.com
}

Genet. Mol. Res. 14 (3): 10322-10330 (2015)

Received February 5, 2015

Accepted May 15, 2015

Published August 28, 2015

DOI http://dx.doi.org/10.4238/2015.August.28.18

\begin{abstract}
Osteoporosis is a common disease characterized by low bone mineral density, deterioration in bone microarchitecture, and increased fracture risk and is more prevalent in postmenopausal women. HLA is a complex gene family; previous studies have shown that it plays an important role in the pathogenesis of osteoporosis among Japanese and Greek populations. Prompted by these findings, this study was designed to explore the associations between $H L A-A$ gene polymorphisms and postmenopausal osteoporosis in the Han Chinese population. The polymerase chain reaction-sequence-based typing method was used for DNA genotyping at the $H L A-A$ locus in 70 patients with postmenopausal osteoporosis and 73 healthy controls.
\end{abstract}


We identified 17 HLA-A alleles in patients with postmenopausal osteoporosis and $20 H L A-A$ alleles in control subjects. Furthermore, we found that the frequency of the $H L A-A^{*} 02: 07$ allele was significantly higher in patients with postmenopausal osteoporosis than in control subjects $(\mathrm{P}=0.023)$, and the relative risk was $4.065(95 \%$ confidence interval $=1.109-14.893$ ). Our study provides supportive evidence for the contribution of $H L A-A$ gene polymorphisms to the susceptibility to postmenopausal osteoporosis and suggests that $H L A-A^{*} 02: 07$ is likely an important genetic risk factor for postmenopausal osteoporosis in the Han Chinese population.

Key words: Human leukocyte antigen-A; Polymorphisms; Polymerase chain reaction-sequence-based typing; Postmenopausal osteoporosis; Bone mineral density

\section{INTRODUCTION}

Osteoporosis is a major public health problem in rapidly aging populations, especially in postmenopausal women (Pietschmann et al., 2009; Esfahanian et al., 2012; Gammage et al., 2012; Mohammadi et al., 2014). It has been proven that osteoporosis demonstrates distinct age and gender characteristics (Pietschmann et al., 2009; Gammage et al., 2012). This systemic skeletal disease is characterized by reduced bone mass and microarchitectural deterioration of the bone tissue, which give rise to increased bone fragility and susceptibility to nontraumatic fractures (Dodd and Rowe, 2013). Osteoporosis-induced fractures further lead to serious consequences such as an increased risk of death, long-term nursing home care, or permanent limitations in mobility and performance of daily living activities (Esfahanian et al., 2012; Lai et al., 2013; Yang et al., 2013). Although osteoporosis has been studied for decades, its exact etiology and pathogenic mechanisms remain inconclusive (Martínez-Maestre et al., 2013).

To date, a number of genes have been shown to be associated with bone mineral density (BMD), and many studies have proven that genetic factors play an important role in the pathogenesis of osteoporosis (Prockop, 1998; Theoleyre et al., 2004; Özbaş et al., 2012; Yang et al., 2013; Athanasiadis et al., 2014; Boroňová et al., 2014; Mohammadi et al., 2014; Oei et al., 2014). The human leukocyte antigen (HLA) system is the most polymorphic immunogenetic system and is located on chromosome 6p21.3 (Schreuder et al., 2005). Currently, more than 10,246 alleles have been identified in the HLA system (IMGT/HLA Database 3.18) (http://www.ebi.ac.uk/imgt/hla/stats.html). The high degree of polymorphism of the HLA system has made it valuable for disease-association studies, which have linked $H L A$ alleles to susceptibility to more than 100 diseases (Carrington and O'Brien, 2003; Noble et al., 2010; Zhou et al., 2011).

Recently, the HLA system has been demonstrated to be involved in several bone metabolic disorders such as rheumatoid arthritis and ankylosing spondylitis (Vignal et al., 2009; Cauli et al., 2013; Cojocaru and Chicoş, 2013; Djidjik et al., 2014). Furthermore, several studies have demonstrated strong associations between certain HLA alleles and osteoporosis susceptibility in the Japanese and Greek female populations (Tsuji et al., 1998; Douroudis et al., 2007). For instance, Tsuji et al. (1998) investigated the association of HLA polymorphism with peak bone mass (PBM) in order to elucidate the genetic background of bone metabo- 
lism in young Japanese women. They concluded that the $H L A-A^{*} 24-B^{*} 07-D R B^{*} 01$ haplotype might be a new genetic marker implicated with low PBM in healthy young Japanese women. In addition, Douroudis et al. (2007) found that $H L A-B 7,-D R 15$, and $-D Q 6(\mathrm{P}=0.026)$ were associated with a lower BMD measured at the forearm; their study showed a significant association between $H L A$ alleles and bone mass loss in postmenopausal osteoporosis within the Greek population. However, to the best of our knowledge, there are few studies concerning the association of $H L A$ gene polymorphisms and osteoporosis susceptibility in the Han Chinese population. We previously investigated the relationship between $H L A-B$ gene polymorphisms and postmenopausal osteoporosis in the Han Chinese population and the results showed that $H L A-B^{*}$ 35:01 was likely an important genetic risk factor for postmenopausal osteoporosis susceptibility (Li et al., 2014). Therefore, the current study was designed to further explore the possible contribution of the HLA-A gene, another major HLA gene in the class I locus, to postmenopausal osteoporosis in the Han Chinese population.

\section{MATERIAL AND METHODS}

\section{Subjects}

The protocol of this study was approved by the Institutional Medical Ethics Committee of Xi'an Jiaotong University. Written informed consent was obtained from all subjects. Participants were random unrelated women of Han Chinese ethnicity whose ancestors had lived in the Shaanxi Province for at least three generations.

Each subject was examined clinically and detailed information such as age, menopause, and history of osteoporotic fracture were collected. Women with a history of bone disease, metabolic or endocrine disorders such as diabetes mellitus, hyperthyroidism, and any systemic illness known to affect bone metabolism were excluded from the study. In addition, women taking drugs that might influence bone metabolism, including calcium supplements, or who were under treatment with drugs known to affect BMD were also ruled out from the study. In total, 70 women (aged 50-65 years) with primary postmenopausal osteoporosis and 73 healthy aged-matched women (aged 51-65 years) were recruited.

\section{Bone densitometry}

Dual-energy X-ray absorptiometry (Lunar Corp., Madison, WI, USA) was used to measure BMD at the lumbar spine (L1-L4) and femoral neck by two qualified radiologists who were blinded to other medical data. BMD is reported was expressed in grams per square centimeter $\left(\mathrm{g} / \mathrm{cm}^{2}\right)$ and as peak bone mass percentage in normal subjects (T-score). According to the criteria of the World Health Organization, osteoporosis in postmenopausal women was diagnosed when the T-score $<-2.5$ standard deviations.

\section{Peripheral blood DNA extraction}

For each subject, $2 \mathrm{~mL}$ peripheral venous blood was drawn into a sterile tube containing the anticoagulant ethylenediaminetetraacetic acid (EDTA) and stored at $-20^{\circ} \mathrm{C}$ prior to DNA extraction. Genomic DNA was extracted and purified from peripheral blood leukocytes according to the manufacturer protocol (A004-1; Dinguo, Beijing, China). 


\section{$H L A-A$ genotyping}

The polymerase chain reaction-sequence-based typing (PCR-SBT) method was used for genotyping the $H L A-A$ gene. As previously described (Zhou et al., 2012), PCR amplifications were accomplished on a GeneAmp PCR system 9700 system (Applied Biosystems, Foster City, CA, USA); then, amplified DNA fragments were purified and sequenced using ABI PRISM BigDye Terminator Cycle Sequencing Ready Reaction Kits (Applied Biosystems) in an ABI 3730XL DNA Sequencer (Applied Biosystems) according to manufacturer instructions. Finally, genotypes were resolved to four digits according to the updated IMGT/HLA database (3.15.0).

\section{Statistical analysis}

Hardy-Weinberg equilibrium (HWE) for the $H L A$ - $A$ locus was estimated using the Arlequin software package version 3.5 (Laurent Excoffier, CMPG, Zoological Institute, University of Bern, Switzerland). Additionally, genetic parameters, including homozygotes (Hom), heterozygotes (Het), power of discrimination (PD), polymorphism information content (PIC), and probability of paternity exclusion (PPE), were assessed using the PowerStat Version 1.2 spreadsheet (Promega Corporation, Madison, WI, USA). In addition, the allele frequencies of the HLA-A locus were calculated by direct counting using the SPSS13.0 software (SPSS Inc., Chicago, IL, USA).

Finally, genotypic associations related to the case-control study were analyzed using SPSS13.0. The frequencies of the $H L A-A$ alleles were compared between controls and patients using the chi-square test or the Fisher exact test when the expected numbers were less than 5 . Furthermore, odds ratios (ORs) and 95\% confidence intervals (CIs) were also calculated using Cornfield's approximation. $\mathrm{P}<0.05$ was regarded as statistically significant.

\section{RESULTS}

\section{HWE}

Genotyping data from 70 women with primary postmenopausal osteoporosis and 73 healthy controls were included for analysis and no one was excluded due to uninterpretable or missing data.

The alleles in the HLA-A locus were in compliance with HWE in both the postmenopausal osteoporosis group $(\mathrm{P}=0.93239066)$ and in the controls $(\mathrm{P}=0.35612644)$.

\section{Genetic parameters}

Hom, Het, PD, PIC, and PPE are genetic parameters for evaluating the polymorphisms in a gene. A gene locus is considered highly polymorphic when its PD value is higher than 0.8 , its PIC is higher than 0.5 , or its PPE value is higher than 0.5 . As shown in Table 1 , the PD, PIC and PPE values were higher than the cutoff values mentioned above in both patients with postmenopausal osteoporosis and in controls. Therefore, the $H L A-A$ locus was highly polymorphic in both patient and control groups. 
Table 1. Genetic parameters of the $H L A-A$ locus in postmenopausal osteoporosis patients and controls.

\begin{tabular}{llccccc}
\hline Locus & Group & $H_{\mathrm{O}}$ & $H_{\mathrm{E}}$ & PD & PIC & PPE \\
\hline HLA-A & Patients & $10.0 \%$ & $90.0 \%$ & 0.969 & 0.88 & 0.795 \\
& Controls & $12.7 \%$ & $87.3 \%$ & 0.971 & 0.89 & 0.741 \\
\hline
\end{tabular}

$H_{\mathrm{O}}$ : homozygotes; $H_{\mathrm{E}}$ : heterozygotes; PD: power of discrimination; PIC: polymorphism information content; PPE: probability of paternity exclusion.

\section{$H L A-A$ allele frequencies}

Collectively, we found 17 high-resolution $H L A$ - $A$ alleles in 70 patients with postmenopausal osteoporosis. The high-resolution genotyping distribution frequencies of the HLA-A locus are summarized in Table 2. The nine most common alleles with a frequency greater than 5\% were $H L A-A^{*} 11: 01$ (20.7\%), $A^{*} 02: 01(11.4 \%), A^{*} 24: 02(11.4 \%)$, $A^{* 02: 06}$ (7.9\%), $A^{* 02: 07}$ (7.9\%), $\mathrm{A} * 30: 01$ (7.1\%), $\mathrm{A}^{* 03: 01}(6.4 \%), \mathrm{A}^{* 32: 01}(5 \%)$, A*33:03 (5\%).

On the other hand, we detected $20 H L A-A$ alleles in 73 control subjects (Table 2). HLA-A 11:01 (17.8\%) was the most frequent allele, followed by $A^{*} 02: 01$ (16.4\%), $A^{*} 24: 02$ (13.7\%), $A^{*} 01: 01$ (8.9\%), $A^{*} 03: 01$ (5.5\%), $A^{*} 30: 01$ (5.5\%), $A^{*} 31: 01$ (5.5\%), and $A^{*} 33: 03$ $(5.5 \%)$.

Table 2. High-resolution genotyping distributions of $H L A-A$ alleles in postmenopausal osteoporosis patients and controls.

\begin{tabular}{|c|c|c|c|c|c|c|}
\hline \multirow[t]{2}{*}{ HLA-A } & \multicolumn{2}{|c|}{ Controls $(\mathrm{N}=146$ alleles $)$} & \multicolumn{2}{|c|}{ Patients $(\mathrm{N}=140$ alleles $)$} & \multirow[t]{2}{*}{$P$} & \multirow[t]{2}{*}{ OR $(95 \% \mathrm{CI})$} \\
\hline & $\mathrm{N}$ & $\overline{\mathrm{AF}(\%)}$ & $\mathrm{N}$ & $\overline{\mathrm{AF}(\%)}$ & & \\
\hline $\mathrm{A}^{*} 01: 01$ & 13 & 8.9 & 6 & 4.3 & 0.117 & $0.458(0.169-1.114)$ \\
\hline $\mathrm{A}^{*} 02: 01$ & 24 & 16.4 & 16 & 11.4 & 0.222 & $0.659(0.332-1.295)$ \\
\hline$A * 02: 03$ & 4 & 2.7 & 0 & 0 & - & - \\
\hline $\mathrm{A} * 02: 05$ & 1 & 0.7 & 0 & 0 & - & - \\
\hline$A * 02: 06$ & 6 & 4.1 & 11 & 7.9 & 0.180 & $1.990(0.715-5.535)$ \\
\hline $\mathrm{A}^{*} 02: 07$ & 3 & 2.1 & 11 & 7.9 & $0.023^{*}$ & $4.065(1.109-14.893)$ \\
\hline$A^{*} 02: 11$ & 1 & 0.7 & 0 & 0 & - & - \\
\hline$A * 02: 17$ & 1 & 0.7 & 1 & 0.7 & 0.976 & $1.043(0.065-16.841)$ \\
\hline$A * 03: 01$ & 8 & 5.5 & 9 & 6.4 & 0.734 & $1.185(0.444-3.164)$ \\
\hline$A * 03: 02$ & 0 & 0 & 1 & 0.7 & - & - \\
\hline$A^{*} 11: 01$ & 26 & 17.8 & 29 & 20.7 & 0.533 & $1.206(0.669-2.173)$ \\
\hline$A^{*} 11: 02$ & 4 & 2.7 & 2 & 1.4 & 0.439 & $0.514(0.093-2.855)$ \\
\hline$A * 24: 02$ & 20 & 13.7 & 16 & 11.4 & 0.563 & $0.813(0.403-1.641)$ \\
\hline$A * 24: 04$ & 0 & 0 & 1 & 0.7 & - & - \\
\hline A*26:01 & 4 & 2.7 & 6 & 4.3 & 0.477 & $1.590(0.439-5.757)$ \\
\hline$A * 29: 01$ & 1 & 0.7 & 0 & 0 & - & - \\
\hline A*30:01 & $\begin{array}{l}1 \\
8\end{array}$ & 5.5 & 10 & 7.1 & 0.583 & $1.308(0.501-3.416)$ \\
\hline$A * 31: 01$ & 8 & 5.5 & 6 & 4.3 & 0.621 & $0.761(0.257-2.253)$ \\
\hline$A * 32: 01$ & 3 & 2.1 & 7 & 5 & 0.437 & $1.765(0.414-7.530)$ \\
\hline A*33:01 & 1 & 0.7 & 0 & 0 & - & 1.700(0.71 \\
\hline$A * 33: 03$ & 8 & 5.5 & 7 & 5 & 0.812 & $0.881(0.311-2.499)$ \\
\hline A*68:01 & 2 & 1.4 & 1 & 0.7 & 0.586 & $0.518(0.046-5.777)$ \\
\hline
\end{tabular}

AF: allele frequency; OR: odds ratio; CI: confidence interval; $* \mathrm{P}<0.05$.

\section{Association of $H L A-A$ alleles with postmenopausal osteoporosis}

The comparison of sequence-based high-resolution HLA-A genotype distributions between the patients with postmenopausal osteoporosis and controls is presented in Table 2. As is 
shown, the frequency of the $H L A-A^{*}$ 02:07 allele was significant higher in patients with osteoporosis than in the control group $(\mathrm{P}=0.023)$; the relative risk was $4.065(95 \% \mathrm{CI}=1.109-14.893)$.

Most previous association studies of $H L A-A$ and bone-related diseases were based on low-resolution (serotypes) of the $H L A$ gene. To be compatible with previous studies, we grouped our sequence-based high-resolution HLA genotypes to their associated serotypes according to the classification of Holdsworth et al. (2009). As for $A 2, A 24$ was also reported to be associated with bone diseases in previous populations; we therefore considered $A 2$ and $A 24$ as candidate alleles in this study. Our results disclosed that no significant increase of $H L A-A^{*} 24$ allelic frequency was identified in the patient group when compared with controls, with OR $=0.871(95 \% \mathrm{CI}=0.436-1.741 ; \mathrm{P}=0.695)$ (Table 3$)$. Furthermore, there was also no significant difference in $H L A-A^{*} 2$ allelic frequency between patients and controls, with $\mathrm{OR}=1.023$ $(95 \% \mathrm{CI}=0.609-1.719 ; \mathrm{P}=0.931)($ Table 3$)$.

\begin{tabular}{|c|c|c|c|c|c|}
\hline Serotype & DNA-based genotype & Controls ( $\mathrm{N}=146$ alleles) & Patients ( $\mathrm{N}=140$ alleles $)$ & $\mathrm{P}$ & OR $(95 \% \mathrm{CI})$ \\
\hline $\mathrm{A} * 2$ & $\begin{array}{l}\mathrm{A}^{*} 02: 01 \\
\mathrm{~A}^{*} 02: 03 \\
\mathrm{~A}^{*} 02: 05 \\
\mathrm{~A}^{*} 02: 06 \\
\mathrm{~A}^{*} 02: 07 \\
\mathrm{~A}^{*} 02: 11 \\
\mathrm{~A}^{*} 02: 17\end{array}$ & $40(27.4 \%)$ & $39(27.9 \%)$ & 0.931 & $1.023(0.609-1.719)$ \\
\hline $\mathrm{A} * 24$ & $\begin{array}{l}\mathrm{A}^{* 24: 02} \\
\mathrm{~A} * 24: 04\end{array}$ & $20(13.7 \%)$ & $17(12.1 \%)$ & 0.695 & $0.871(0.436-1.741)$ \\
\hline
\end{tabular}

OR: odds ratio; CI: confidence interval.

\section{DISCUSSION}

Osteoporosis, like other genetically complex diseases, is the product of the interactions between genetic and environmental factors (Yang et al., 2013; Boroňová et al., 2014). Several studies have proven that genetic factors might play an important role in the pathogenesis of osteoporosis (Prockop, 1998; Theoleyre et al., 2004; Özbaş et al., 2012; Yang et al., 2013; Athanasiadis et al., 2014; Boroňová et al., 2014; Mohammadi et al., 2014; Oei et al., 2014). With the rapid development of genome-wide association studies, an increasing number of osteoporosis susceptibility genes are expected to be reported. However, their candidacy will require further evidence from different populations (Yang et al., 2013). The purpose of the present study was to investigate the relationships between $H L A-A$ gene polymorphisms and postmenopausal osteoporosis susceptibility in the Han Chinese population.

Our study is the first to explore the associations between $H L A-A$ gene polymorphisms and postmenopausal osteoporosis in the Han Chinese population using the PCR-SBT method. HLA-A is a group of HLA that are encoded by the HLA-A locus, which is located at human chromosome 6p21.3. HLA-A is ranked among the genes in humans with the fastest-evolving coding sequence; the concomitant variation promotes genetic diversity in the population. However, an important clinical risk factor in the pathogenesis of osteoporosis is the presence of genetic polymorphism in and around susceptibility genes and regions. The association between HLA-A antigens and bone metabolic disorders has also been investigated in several populations. For example, Huang et al. (2007) found that the frequency of the $H L A-A^{*} 02: 03$ 
allele was significantly higher in patients with osteoarthritis than in controls. In addition, Tsuji et al. (1998) concluded that the $H L A-A^{*} 24$ allele frequency was high in patients with low PBM and that the HLA-A*24-B*07-DRB*01 haplotype could be considered a new genetic marker associated with low PBM in healthy young Japanese women. However, we did not detect significant differences of $H L A-A^{*} 24$ or $H L A-A^{*} 02: 03$ allele frequencies between patients and controls in our study population. Nevertheless, we found that the frequency of the $H L A-A^{*}$ 02:07 allele was significantly higher in patients with postmenopausal osteoporosis than in the control group and the relative risk was $4.065(95 \% \mathrm{CI}=1.109-14.893)$. Our results present strong evidence that $H L A-A^{*}$ 02:07 might be involved in osteoporosis susceptibility, indicating that $H L A-A^{*}$ 02:07 might be a major risk gene of postmenopausal osteoporosis in Han Chinese population. We speculated that the conflicting results between different populations might be attributed to differences in sample size, HLA typing methodology, ethnic background, and geographic variations. These could be due to the fact that the distributions of $H L A$ alleles and haplotypes show high variation in different ethnic groups or even within the same ethnic group living in different geographical areas (Zhou et al., 2011).

Bone homeostasis is maintained by a balance between bone resorption by osteoclasts and bone formation by osteoblasts. This process is regulated by the immune system and its imbalance often results in osteoporosis (Tanaka, 2013; Jianbo et al., 2014). Increasing evidence suggests that immunological factors play an important role in the pathogenesis of osteoporosis. Molnár et al. (2014) found that increased levels of IL-17A, which plays an important role in the bone-resorption process, are involved in postmenopausal osteoporosis. Additionally, Zhao (2013) reported that T-helper 17 cells are critical modulators in the pathogenesis of estrogen-deficient osteoporosis and that therapeutic strategies targeting IL-17 networks might be clinically useful in the treatment of postmenopausal osteoporosis. The study of Kim et al. (2010) indicated that leukocyte common antigen-related (LAR) tyrosine phosphatase positively regulates osteoblast differentiation by modulating extracellular signal-regulated kinase activation. LAR phosphatase could be used as a novel regulatory target protein in many bone-associated diseases including osteoporosis. $H L A-A$ is one of the most important immunoregulatory genes, and our results further support the view that polymorphisms in the $H L A-A$ gene might further influence immune system involvement in regulating bone homeostasis (Benasciutti et al., 2014).

Our study has several strengths: 1) Ethnic background and geographic variations are extremely important factors affecting study results. China has 56 officially identified ethnic groups, and the Han nationality constitutes approximately $92 \%$ of the Chinese population. In the present study, we selected subjects from the same ethnic (Han nationality) and geographic backgrounds (Shaanxi Province) to avoid the possible occurrence of selection bias. 2) Women with conditions potentially causing secondary osteoporosis and women over the age of 65 years were ruled out from the present study to avoid the impact of senile osteoporosis. 3) Samples were genotyped at random in the same laboratory. This is important for avoiding the potential biases that can arise when samples are genotyped at different centers. 4) All samples in the patient and control groups were in HWE at the HLA-A locus. In addition, we examined Hom, Het, PD, PIC, and PPE, which are genetic parameters for evaluating the polymorphisms in a gene. A gene locus is considered highly polymorphic when its PIC is higher than 0.5 , its PD value is higher than 0.8 , or its PPE value is higher than 0.5 . In our present study, the PIC, $\mathrm{PD}$, and PPE values were higher than the respective cutoff values. Therefore, the $H L A-A$ data obtained from our sample population were highly polymorphic, which is valuable for further 
genetic research. 5) The PCR-SBT method was used to examine the associations between postmenopausal osteoporosis and $H L A$ alleles. The PCR-SBT method is considered the gold standard for high-resolution definition of $H L A$ alleles (Woo et al., 2012). Previous HLA association studies in osteoporosis were performed with low-resolution $H L A$ serologic typing by the PCR-sequence specific primers (PCR-SSP) method. The PCR-SBT method has much higher accuracy and reliability than simple serological typing methods, and also facilitates the standardization of the $H L A$-typing process.

In conclusion, our study provides supporting evidence for the contributions of $H L A-A$ gene polymorphisms to postmenopausal osteoporosis susceptibility in the Han Chinese population. Our results suggest that $H L A-A^{*} 02: 07$ is likely an important risk factor for postmenopausal osteoporosis and that it might play an important role in the pathogenesis of osteoporosis. Given that different populations have different $H L A$ polymorphisms, further investigation of the relationships between various $H L A$ genes and osteoporosis with larger sample size in different ethnic samples is still necessary to confirm our findings.

\section{Conflicts of interest}

The authors declare no conflict of interest.

\section{ACKNOWLEDGMENTS}

Research supported by the National Natural Science Funds of China (\#81273018; \#30700654), the Science Funding of the Health Department, Shaanxi Province (\#2012D58), the Natural Science Funding of Shaanxi Province (\# 2015JM8436), and the Fundamental Research Funds for the Central University (\#XJJ 2011024).

\section{REFERENCES}

Athanasiadis G, Malouf J, Hernandez-Sosa N, Martin-Fernandez L, et al. (2014). Linkage and association analyses using families identified a locus affecting an osteoporosis-related trait. Bone 60: 98-103.

Benasciutti E, Mariani E, Oliva L, Scolari M, et al. (2014). MHC class II transactivator is an in vivo regulator of osteoclast differentiation and bone homeostasis co-opted from adaptive immunity. J. Bone Miner. Res. 29: 290-303.

Boroňová I, Bernasovská J, Mačeková S, Petrejčíková E, et al. (2014). TNFRSF11B gene polymorphisms, bone mineral density, and fractures in Slovak postmenopausal women. J. Appl. Genet. 56: 57-63.

Carrington M and O' Brien SJ (2003). The influence of HLA genotype on AIDS. Annu. Rev. Med. 54: 535-551.

Cauli A, Shaw J, Giles J, Hatano H, et al. (2013). The arthritis-associated HLA-B*27:05 allele forms more cell surface B27 dimer and free heavy chain ligands for KIR3DL2 than $H L A-B * 27: 09$. Rheumatology 52: 1952-1962.

Cojocaru M and Chicoş B (2013). Genetic differences between patients with rheumatoid arthritis. Rom. J. Intern. Med. 51: 89-91.

Djidjik R, Allam I, Douaoui S, Meddour Y, et al. (2014). Association study of human leukocyte antigen-DRBI alleles with rheumatoid arthritis in Algerian patients. Int. J. Rheum. Dis. doi: 10.1111/1756-185X.12272 (Epub ahead of print).

Dodd DZ and Rowe DJ (2013). The relationship between postmenopausal osteoporosis and periodontal disease. J. Dent. Hyg. 87: 336-344.

Douroudis K, Tarassi K, Athanassiades T, Giannakopoulos F, et al. (2007). HLA alleles as predisposal factors for postmenopausal osteoporosis in a Greek population. Tissue Antigens 69: 592-596.

Esfahanian V, Shamami MS and Shamami MS (2012). Relationship between osteoporosis and periodontal disease: review of the literature. J. Dent. 9: 256-264.

Gammage KL, Gasparotto J, Mack DE and Klentrou P (2012). Gender differences in osteoporosis health beliefs and knowledge and their relation to vigorous physical activity in university students. J. Am. Coll. Health 60: 58-64. 
Holdsworth R, Hurley CK, Marsh SG, Lau M, et al. (2009). The HLA dictionary 2008: a summary of $H L A-A,-B,-C$, $-D R B 1 / 3 / 4 / 5$, and $-D Q B 1$ alleles and their association with serologically defined HLA-A, -B, -C, -DR, and -DQ antigens. Tissue Antigens 73: 95-170.

Huang LH, Wang JB, Wu H and Sun XL (2007). Relations between frequency of HLA-A, HLA-DRB and clinical features of osteoarthritis. Chin. J. Lab. Diagn. 11: 1301-1303.

Jianbo L, Zhang H, Yan L, Xie M, et al. (2014). Homocysteine, an additional factor, is linked to osteoporosis in postmenopausal women with type 2 diabetes. J. Bone Miner. Metab. 32: 718-724.

Kim WK, Bae KH, Choi HR, Kim DH, et al. (2010). Leukocyte common antigen-related (LAR) tyrosine phosphatase positively regulates osteoblast differentiation by modulating extracellular signal-regulated kinase (ERK) activation. Mol. Cells 30: 335-340.

Lai CL, Tseng SY, Chen CN, Liao WC, et al. (2013). Effect of 6 months of whole body vibration on lumbar spine bone density in postmenopausal women: a randomized controlled trial. Clin. Interv. Aging 8: 1603-1609.

Li SM, Zhou DX and Liu MY (2014). Associations between polymorphisms of HLA-B gene and postmenopausal osteoporosis in Chinese Han population. Int. J. Immunogenet. 41: 324-329.

Martínez-Maestre MA, Machuca G, González-Cejudo C, Flores JR, et al. (2013). Osteoporosis, fragility fracture, and periodontal disease: a cross-sectional study in Spanish postmenopausal women. Menopause 20: 79-84.

Mohammadi Z, Fayyazbakhsh F, Ebrahimi M, Amoli MM, et al. (2014). Association between vitamin D receptor gene polymorphisms (Fok1 and Bsm1) and osteoporosis: a systematic review. J. Diabetes Metab. Disord. 13: 98.

Molnár I, Bohaty I and Somogyiné-Vári E (2014). IL-17A-mediated sRANK ligand elevation involved in postmenopausal osteoporosis. Osteoporos. Int. 25: 783-786.

Noble JA, Valdes AM, Varney MD, Carison JA, et al. (2010). HLA class I and genetic susceptibility to type 1 diabetes: results from the Type 1 Diabetes Genetics Consortium. Diabetes 59: 2972-2979.

Oei L, Hsu YH, Styrkarsdottir U, Eussen BH, et al. (2014). A genome-wide copy number association study of osteoporotic fractures points to the 6p25.1 locus. J. Med. Genet. 51: 122-131.

Özbaş H, Tutgun Onrat S and Özdamar K (2012). Genetic and environmental factors in human osteoporosis. Mol. Biol. Rep. 39: 11289-11296.

Pietschmann P, Rauner M, Sipos W and Kerschan-Schindl K (2009). Osteoporosis: an age-related and gender-specific disease - a mini-review. Gerontology 55: 3-12.

Prockop DJ (1998). The genetic trail of osteoporosis. N. Engl. J. Med. 338: 1061-1062.

Schreuder GM, Hurley CK, Marsh SG, Lau K, et al. (2005). HLA dictionary 2004: summary of HLA-A, -B, $-C,-D R B 1$ alleles and their association with serologically defined HLA-A, -B, -C, -DR, and -DQ antigens. Hum. Immunol. 66: $170-210$.

Tanaka Y (2013). Secondary osteoporosis or secondary contributors to bone loss in fracture. The relevance of immune system to bone metabolism. Clin. Calcium 23: 1265-1270.

Theoleyre S, Wittrant Y, Tat SK, Fortun Y, et al. (2004). The molecular triad OPG/RANK/RANKL: involvement in the orchestration of pathophysiological bone remodeling. Cytokine Growth Factor Rev. 15: 457-475.

Tsuji S, Munkhbat B, Hagihara M, Tsuritani I, et al. (1998). HLA-A*24- $B^{*} 07-D R B 1 * 01$ haplotype implicated with genetic disposition of peak bone mass in healthy young Japanese women. Hum. Immunol. 59: 243-249.

Vignal C, Bansal AT, Balding DJ, Binks MH, et al. (2009). Genetics association of the major histocompatibility complex with rheumatoid arthritis implicates two non-DRB1 loci. Arthritis Rheum. 60: 53-62.

Woo HI, Joo EY, Hong SB, Lee KW, et al. (2012). Use of PCR with sequence-specific primers for high-resolution human leukocyte antigen typing of patients with narcolepsy. Ann. Lab. Med. 32: 57-65.

Yang H, Zhang B, Zhu J, Liu D, et al. (2013). 4q22.1 contributes to bone mineral density and osteoporosis susceptibility in postmenopausal women of Chinese Han population. PLoS One 8: e80165.

Zhao R (2013). Immune regulation of bone loss by Th17 cells in oestrogen-deficient osteoporosis. Eur. J. Clin. Invest. 43: 1195-1202.

Zhou DX, Zhang J, Wang HX, Wang XF, et al. (2011). Association study of HLA-B alleles with idiopathic male infertility in Han population of China. J. Assist. Reprod. Genet. 28: 979-985.

Zhou DX, Huang XC, Wang XF, Zhang J, et al. (2012). Association study of human leukocyte antigen-A gene with idiopathic male infertility in Han population of China. Andrologia 44: 213-218. 